\title{
Paraganglioma gangliocítico duodenal
}

\section{Duodenal gangliocytic paraganglioma}

Jorge Feres Junior ${ }^{1}$; Eduardo Henrique de Freitas Ramos Filho²; Fábio Porto Silveira ${ }^{1}$; Fábio Silveira ${ }^{1}$; Thiago Quirino Tubone ${ }^{3}$

\section{INTRODUÇÃO}

$\mathrm{P}$ araganglioma gangliocítico é um tumor duodenal benigno extremamente raro. Localiza-se geralmente na segunda porção do duodeno próximo à ampola de Vater. Já foram relatados cerca de 130 casos na literatura mundial. Na maioria dos casos o diagnóstico foi realizado após duodenopancreatectomia, haja vista a dificuldade do diagnóstico histológico pré-operatório, por se tratar de um tumor submucoso 4 .

Apresenta-se com diferentes padrões histológicos, podendo ser semelhante a neurofibroma, ganglioneuroma ou tumor carcinóide ${ }^{5}$. Estudo imunoistoquímico pode ser realizado para ratificação e identificação do tipo histológico.

\section{RELATO DO CASO}

Paciente do sexo masculino, 42 anos, procura atendimento médico devido à dor abdominal difusa tipo cólica há aproximadamente um mês com piora importante na última semana e associada à icterícia progressiva, colúria e hipocolia fecal. Referia também um episódio prévio de melena antes de tornar-se ictérico e emagrecimento de 3 $\mathrm{Kg}$ em um período de três meses.

O paciente encontrava-se em regular estado geral, ictérico $+2 /+4$, pressão arterial de $140 / 90 \mathrm{mmHg}$, afebril. A ausculta cardiopulmonar mostrou-se sem alterações, bem como o exame abdominal.

Laboratorialmente apresentava bilirrubinas aumentadas (direta:5,44 e indireta:1,35 mg/dl ), fosfatase alcalina de $272 \mathrm{U} / \mathrm{l}$ e ggt de 519U/l.

Ecograficamente apresentou colelitíase e coledocolitíase em terço distal com dilatação de colédoco proximal. Optou-se então por CPRE que evidenciou à duodenoscopia lesão elevada de $25 \mathrm{~mm}$ de diâmetro na região da papila de Vater e após cateterização do óstio da papila e injeção de contraste: via biliar extra-hepática contrastada parcialmente com imagem sugestiva de cálculo em colédoco (Figura 1). Devido à lesão elevada e à dificuldade da cateterização profunda do colédoco não foi realizado papilotomia e extração do cálculo. A biópsia da lesão evidenciou mucosa duodenal sem alterações.
Após resultado de CPRE, solicitou-se colangioressonância com o seguinte resultado: formação nodular compatível com processo neoplásico na topografia da papila duodenal medindo cerca de $3 \times 4 \times 6 \mathrm{~cm}$ (A-P , L$\mathrm{L}, \mathrm{C}-\mathrm{C}$ ), sem plano de clivagem satisfatório com a cabeça do pâncreas promovendo redução da luz do colédoco distal (infiltração) e litíase no colédoco distal (Figura 2).

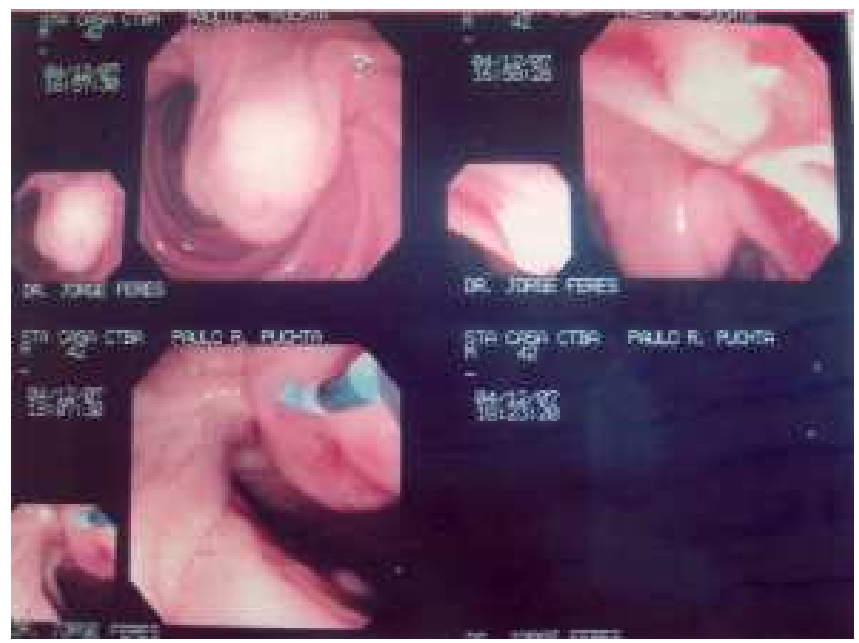

Figura 1 - Lesão periampular dificultando a cateterização do óstio da papila de Vater.

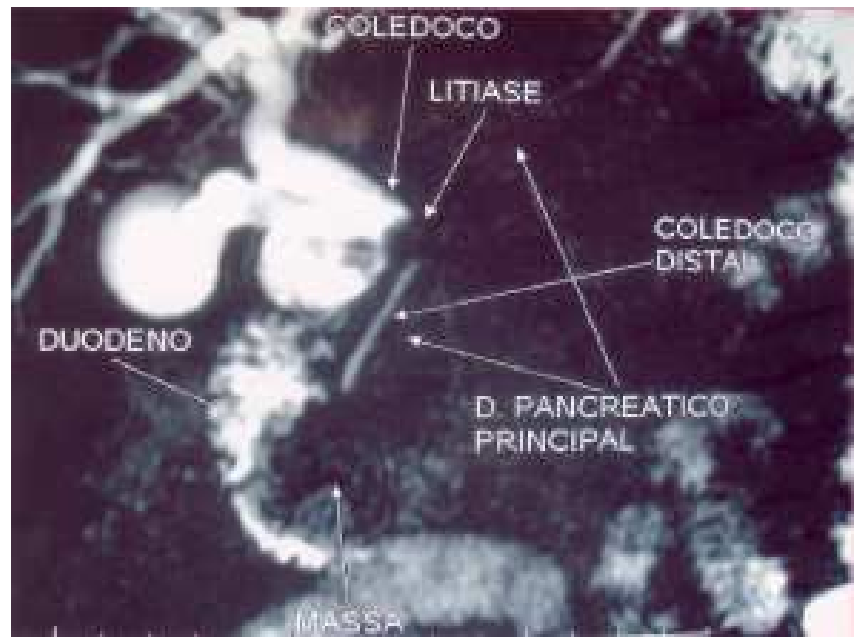

Figura 2 - Lesão tumoral periampular sem plano de clivagem com o pâncreas.

Trabalho realizado na Irmandade de Santa Casa de Misericórdia de Curitiba-PR-BR.

1. Médico Cirurgião da Irmandade Santa Casa de Misericórdia de Curitiba-PR-BR; 2. Médico Endoscopista do Centro de Endoscopia Digestiva de Curitiba (CEDIC)- PR-BR; ${ }^{3}$. Médico Cirurgião Geral formado pela Irmandade Santa Casa de Misericórdia de Curitiba-PR-BR. 
O paciente foi, então, submetido à duodenopancreatectomia. O estudo anatomopatológico da peça ressecada sugeriu a possibilidade de paraganglioma glangliocítico duodenal, necessitando de imunoistosquímica para confirmar o diagnóstico. O estudo imunoistoquímico foi positivo para os anticorpos: CK AE1/AE3, Cromogranina, Sinaptofisina, Ki 67 e S100, confirmando o diagnóstico de paraganglioma.

\section{DISCUSSÃO}

Paraganglioma gangliocítico duodenal é um raro tumor que tende a desenvolver-se na papila ou em suas adjacências. Dahl et al. em 1957 descreveu o primeiro caso ${ }^{6}$. O tumor é duas vezes mais freqüente em homens do que em mulheres e a incidência maior entre 50-60 anos. Seu tamanho varia de 0,5 a $4 \mathrm{~cm}$, com média de $2,5 \mathrm{~cm}$, sendo geralmente único ${ }^{1}$.

Os sinais e sintomas dependem da localização anatômica e incluem dor abdominal, sangramento gastrointestinal, icterícia e obstrução. Não é raro descobrir incidentalmente lesão assintomática.

Devido sua localização submucosa, o diagnóstico histopatológico através de biópsias por endoscopia é raro, sendo diagnosticado habitualmente por estudo da peça cirúrgica.

Histologicamente, o tumor apresenta três tipos celulares que são: células epitelióides, fusiformes e ganglionares. Para o diagnóstico é necessário a presença dos três tipos, quer seja por anatomopatológico convencional ou por estudo imunoistoquímico.

Imunoistoquimicamente, células epitelióides são positivas para NSE, polipeptideo pancreático, somatostatina, cromogranina e sinaptofisina. Células ganglionares são positivas para NSE, sinaptofisina e neurofilamentos. Já as células fusiformes são fortemente positivas para proteína S1007.

A evolução natural do tumor é incerta. Habitualmente apresenta características benignas, porém há casos descritos de metástase para linfonodos regionais. O tratamento do paranganglioma deve ser feito com a ressecção cirúrgica ou endoscópica da lesão.

\title{
A $B$ B S T R A $R$
}

\begin{abstract}
We present a case of a 42-year-old man with abdominal pain, obstructive jaundice and a mass in the second portion of the duodenum, near the papillary region. The patient was operated and the histological study of the specimen returned duodenal gangliocytic paraganglioma (a rare submucosal benign tumor of the duodenum). We also describe, after revision of the literature, the pathology, the diagnosis and its treatment.
\end{abstract}

Key words: Paraganglioma/diagnosis. Carcinoid tumor. Duodenal neoplasms. Ampulla of Vater. Pancreaticoduodenectomy.

\section{REFERÊNCIAS}

1. Prieto C, Zozaya J.M, Iñarrairaegui M, Nantes O, Vila JJ, Beloqui R Duodenal gangliocytic paranganglioma. An Sist Sanit Navar 2005; 28 (1):109-13.

2. Nagai T, Torishima R, Nakashima H, Tanahashi J, Iwata M, Ookawara $\mathrm{H}$, et al. Duodenal gangliocytic paraganglioma treated with endoscopic hemostasis and resection. J Gastroenterol 2004; 39(3):277-83

3. Sakhuja P, Malhotra V, Gondal R, Dutt N, Choudhary A Periampullary gangliocytic paraganglioma. J Clin Gastroentero 2001; 33(2):154-6

4. Dahl EV, Waugh JM, Dahlin DC. Gastrointestinal ganglioneuromas; brief review with report of a duodenal ganglioneuroma. Am J Pathol 1957: 33(5):953-65.
5. Plaza JA, Vitellas K, Marsh WL Jr. Duodenal gangliocytic paraganglioma: a radiological-pathological correlation 2005 9(3):143-7.

Recebido em 20/02/2007

Aceito para publicação em 30/03/2007

Conflito de interesse: nenhum

Fonte de financiamento: nenhuma

\section{Como citar este artigo:}

Feres Júnior J, Ramos Filho EHF, Silveira FP, Silveira F, Tubone TQ. Paraganglioma gangliocítico duodenal. Rev Col Bras Cir. [periódico na Internet] 2011; 38(4). Disponível em URL: http://www.scielo.br/rcbc

Endereço para correspondência:

Eduardo Henrique de Freitas Ramos Filho

E-mail: efreitasramos@bol.com.br 